\title{
Operation of a Concurrent Design Facility for University Projects
}

\author{
Anton B. Ivanov \\ EPFL Space Center (eSpace) \\ EPFL, PPH334, Station 13 \\ Lausanne, Switzerland \\ +41216936978 \\ anton.ivanov@epfl.ch
}

\author{
Louis Masson, Federico Belloni \\ Swiss Space Center \\ EPFL, PPH338, Station 13 \\ Lausanne, Switzerland \\ +41216936946
}

\begin{abstract}
Concurrent Design is a part of the systems engineering process, which has been widely adopted in space mission design. This paper describes the setting up a facility in a university and will try to answer a question of where the Concurrent Design Facility (CDF) concept can fit into education. Hardware setup, software approaches and knowledge management procedures are discussed. The focus of this work was to create an Integrated Design Model (IDM) for nanosats and microsats. This IDM was used to design various space related projects including a Cubesat missions, suborbital plane and a space telescope. We also discuss how to utilize CDF concept after the design phase for validation and verification. CDF was also used for a number of multidisciplinary projects.
\end{abstract}

\section{TABLE OF Contents}

1. INTRODUCTION . .1

2. BACKGROUND ..........................................................1

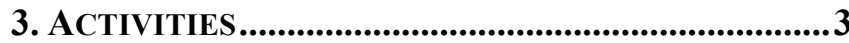

4. BEYOND THE DESIGN PHASE ...............................6

5. Challenges ..............................................................6

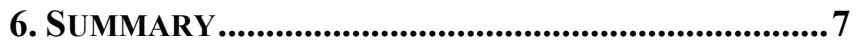

ACKNOWLEDGEMENTS ............................................. 7

REFERENCES .........................................................7 7

BIOGRAPHY ................................................................8

\section{INTRODUCTION}

The EPFL (Ecole Polytechnique Federal de Lausanne) Space Center was created in 2003 to federate needs, resources, and opportunities to strengthen positions of its partners in the Space domain, from an institutional, academic and industrial point of view. The Center's mission has further evolved to promote space related research and development activities at EPFL by providing high-standard education, leading space technology development activities and coordinating multi-disciplinary learning projects

Concurrent Design (or Engineering) Facilities (CDF) exist now in many organizations that are oriented towards projects. CDF at European Space Agency's ESTEC centre [1] or space mission oriented TeamX [2], which was part of the JPL Project Design Center [3], are at the root of these activities. This experience was followed by other space agencies (German Space Agency (DLR), [4]) and

978-1-4673-7676-1/16/\$31.00 C2016 IEEE commercial companies, such as Airbus Defence and Space [5] and $\mathrm{OHB}$ [6]. Academic environment requirements are focused on student education and research projects, as opposed to continuous product development. Basic principles of a CDF, such as team work, integrated design model and concentrated design sessions remain the same. This paper will describe the process of setting up a facility in a university and will try to answer a question of where the CDF concept can fit into a university environment.

\section{BACKGROUND}

\section{Concurrent design facility for a University}

CDF is now recognized as a teaching tool at many universities. The faculty of Aerospace Engineering at Technical University of Delft is using this approach in its course for spacecraft systems engineering (FireSat example, [7]). There are also extensive plans to develop a general purpose, multidisciplinary Concurrent Engineering Design Laboratory (CEDL) at Skoltech, a new private research institution opened in collaboration with MIT in 2011 [8].

CDF Facility at the EPFL Space Center was established in 2007 to support multi-disciplinary research projects. It is used in the curriculum of the minor in space technologies and to support non-space activities. The primary goal for the EPFL CDF described in this paper, is to improve quality of education, to provide complement to system engineering curriculum, and to provide introduction to model based systems engineering for EPFL students from different faculties and options. Currently there are 2 major activities that are present:

- $\quad$ Teaching of the classes offered by the Space Center. These classes immerse students into the process of satellite development and their applications.

- $\quad$ Employment of the Systems Engineering methodology to teach or conduct projects in fields other than space (e.g. Mechanical Engineering, Information Science).

\section{Facility}

The CDF room (shown in Figure 1) contains 10 desktops and 3 workstations. Each subsystem is capable of showing its data on the screen, and the facilitator can switch between various subsystems. There are 3 projectors and 2 
SmartBoards. Interaction with computer and data are done via use of SmartBoards. Facility was upgraded in 2012 to bring it up to date with existing computing technologies.

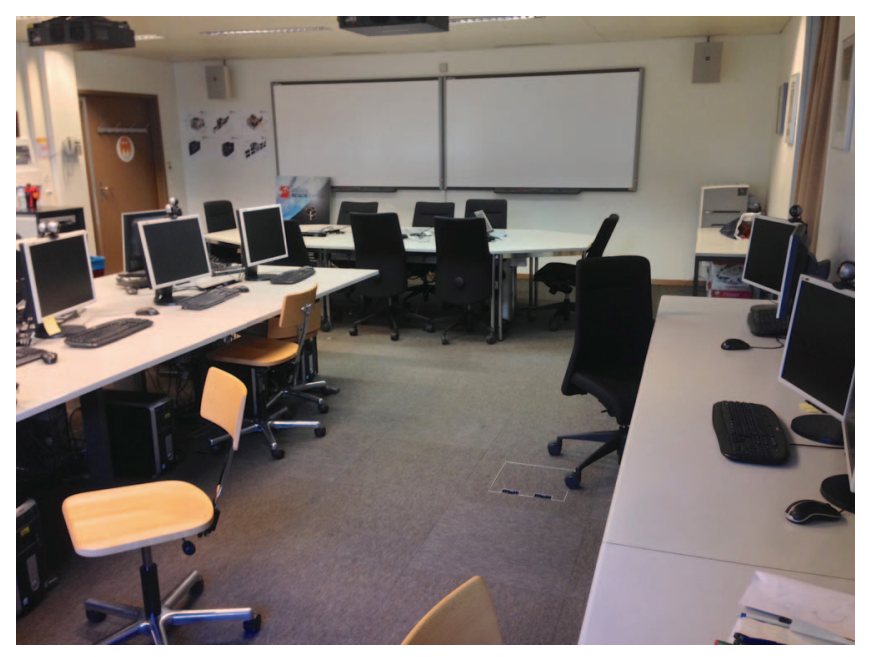

Figure 1 General view of the CD facility at the EPFL Space Center before moving to the new building. Note back-to-back layout of the stations to facilitate conversations during design studies. In the background there are two SmartBoards, which are supported by the ceiling projectors. This setup proved to be very effective for teaching and team meetings.

\section{Software}

The first version of the CDF data exchange and session management software was a set of simple Excel Visual Basic macros, inspired by the ESTEC's CDF-IDM (Integrated Date Model) model. Development and deployment was fast and easy, but it required considerable amounts of maintenance once projects grew in scope and number. In 2011 a decision was taken to change the Excel/database interface with the CDP (Concurrent Design Process) software from the J-CDS company [9] via an academic license. This allowed for much easier maintenance of the database, switching between options and iterations and much more. Existing projects and Excel macros (interface other software packages) were integrated into CDP. In order to address the learning curve for the software, a class was organized at the Center for engineers and students. The system is currently capable of interfacing with STK, MATLAB and CATIA software packages. This capability is needed in order to exchange engineering data between calculations and the CDP database.

Microsoft Excel is the primary user interface. It is known by most of the students or relatively easy to start using. Database details are hidden from users and they are presented with a product tree, which is intuitive and easy to use. Satellite ToolKit (STK, by Analytical Graphics, Inc) is used to obtain all geometry information on relative position of a satellite relative to ground station and the Sun. Information is supplied directly to the Excel worksheet for information exchange. CATIA or Solidworks are used to control configuration of the spacecraft and for bottom up estimation of mass budget and balance (moment of inertia). Data can also be transferred directly into the data structure of the CDF. Open Concurrent Data Tool (OCDT) infrastructure was released recently by the European Space Agency, which will now serve as a baseline for new projects (discussed in the Challenges section).

\section{Implementation approach.}

In order to prepare an initial design of a system, models were developed to describe behaviour of various components (often these models depend on one another for parameters). In addition, databases were populated with components for all subsystems. To follow on a famous quote "algorithms + data = programs" by Wirth [10], in a CDF environment we can propose that

$$
\text { models }+ \text { databases }=\text { system }
$$

\section{Models}

Design models were created for subsystems in Cubesat standard satellites (between 1 and $10 \mathrm{~kg}$ ) and small satellites (up to $250 \mathrm{~kg}$ ). Models are implemented in the following engineering software packages: MATLAB for AOCS (Attitude and Orientation Control System) and payload analysis; CATIA or SolidWorks for mechanical properties; ESATAN/ESARAD or MATLAB Simulations [11] for thermal analysis; AGI STK for mission simulation, power and data budget calculations. Some models scale well across range of satellite classes, e.g. mechanical properties or link budgets. But payload and AOCS model have to be developed for each study.

\section{Databases}

Component databases were populated for all nano and micro satellite subsystems. Databases need to be updated constantly. This assignment fits very well with Master level students, who are starting their minor in Space Technologies.

\section{Team}

CDF relies on students to carry out projects. Students come for either semester project or a master project, which may last for either one semester (14 weeks) or two. The number of students varies from a small team of 4 to 20 . In the latter case students are split into focused teams of 4 to 7 people, depending on their background and academic aptitude. Semester projects are required for all EPFL students starting in the 3rd year. Projects offered by the Space Center are usually taken by Master Level year students. Study leaders are EPFL scientists, doctoral students or young engineers. In most cases students starting their Minor in Space Technologies do not have the team experience necessary for carrying out a project in a group. Therefore they face a steep learning curve. This is a major difference between a CDF facility in an industrial setting (such as ESTEC or JPL or a commercial company) and an educational institution. We cannot train and retain experts for studies (with the exception of doctoral students); on the contrary, experts are 
trained for each and every study. The downside is that studies cannot performed any given study in one week, similar to timescales of industrial CDFs. On the positive side is the fact that CDF in an educational setting can be adapted to a much wider range of projects. Students gain necessary experience to work in a team-oriented fast paced environment.

\section{Process}

The project starts with a Kick-Off meeting, where the goals and status of the project are explained. Students have 2 weeks to prepare a literature review for their first presentation. They research what was published recently and existing reports and technical notes from the project. Once individual goals have been set, the design phase begins to result in a midterm review. Implementation (either software or hardware) usually lasts about a month or longer. The four-month semester is finished with short testing or integration activities. All students are required to document their work, such that it can be continued in the next semester. Reports are discussed and corrected in detail during final presentation. Final reports are archived in the web-based archive system, provided by EPFL (my.epfl).

\section{ACtivities}

Since its inception in early 2007 all of the CDF hardware and software has been set up and operational. The CDF classroom is in operation not only as a concurrent design facility but also as a regular computer class. The Space Center staff and students in all project activities, which are described below, use this class. Since 2010, the team have finished development of a structure to support design of Cubesat-type satellites in concept phase and Phase A. A set of models for microsatellites was developed for a study of an exoplanets observatory satellite. Migration of the database to the infrastructure from J-CDS company provided a solid foundation for all future projects and possible projects with Swiss industry.

\section{Projects}

Concurrent design approach was used in projects related to satellite systems engineering and to general aerospace systems. We have implemented a basic database and models for nanosatellites (SwissCube [11] and CubETH [12]), and a small space telescope for CHaracterisation of ExoPlanet Satellite [13] (CHEOPS). Together with the Interdisciplinary Aerodynamics Group, design study was carried out for a suborbital airplane capable of reaching $100 \mathrm{~km}$ altitude to provide 0 -g experience for customers.

\section{CubETH}

As an example, below is a description of study that was performed in 2012. The goal of the project was to prepare a trade study for a follow-up to SwissCube. The Geodesy lab at ETH Zürich formulated scientific requirements. A Team of 4 students was supervised by a young engineer (team system engineer) and senior staff (team lead). The main question of this study was to create a Cubesat mission, utilizing to the maximum extent possible, design of the SwissCube, while minimizing development and manufacturing time. Since scientific requirements were different, "build-to-print" of previous design could not be used. Key changes were in the requirements for attitude determination and control and significantly higher data rate. In attitude determination and control, the trade was to understand whether the existing system would satisfy new science requirements. On the telecom system the requirement was to support a much higher data rate (9.6 $\mathrm{kbit} / \mathrm{sec}$ instead of $1.2 \mathrm{kbit} / \mathrm{sec})$. Another side to the study was to account for lessons learned from SwissCube and change some elements of design or testing procedures. This project is now being implemented by a consortium of Swiss Federal Universities (ETH) and Universities of Applied Sciences.

Integrated data model for CubeSat type stallites was created at EPFL. It is valid for nanosats between 1 and $12 \mathrm{~kg}$ (1U to $6 \mathrm{U})$. The product tree was moved to the J-CDS structure. Current configuration of the CubETH satellite is shown in Figure 2 (rendering from SolidWorks).

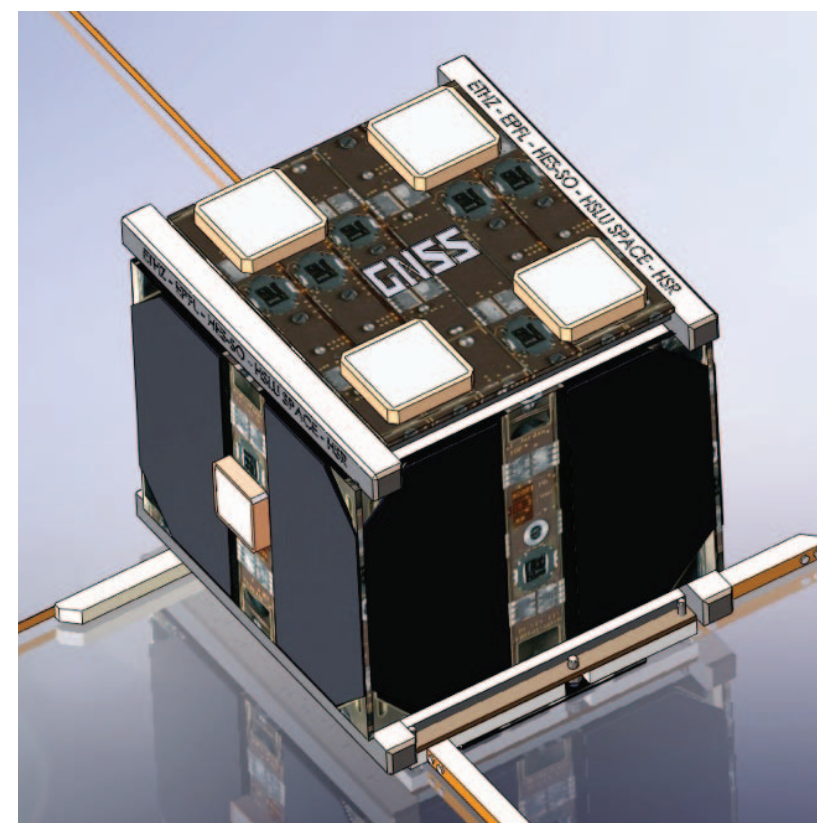

Figure 2 Computer rendering of the CubETH nanosatellite. Five GNSS antennas are situated in the top panel and one side panel. Four UHF and VHF antennas are shown deployed from the Antenna deployment system. This is a standard $1 \mathrm{U}$ Cubesat.

Systems - The main goal was to track trade-offs in different subsystems as well as mass, power and cost budgets. For each of the subsystems a trade-off was created with categories: reuse SwissCube, develop new or buy existing. Particular attention here is paid to tracking and validation of requirements. Due to budgetary constraints, use of a commercial system like DOORS is not possible. Therefore, 
our CDF is employing simple Excel tables and experimenting with freely available tools. Recent versions of J-CDS software include some tools for tracing and validation of requirements. If successful, they will provide a complete solution for systems engineering studies in an academic setting.

It is important to write quality requirements. The project was not able to afford any tools to help with requirements writing. The best method we have found was to meet regularly with the team and discuss at length the text of the requirements. Particular attention was given to use of "shall" and "will" statements and derivation of test cases to provide validation. Since the work was mostly done inhouse, a 3-level requirement model was found to be sufficient to implement the project (Project / System / Subsystem ). Figure 3 shows partial detail of the Product Breakdown structure that was adopted for the CubETH project.

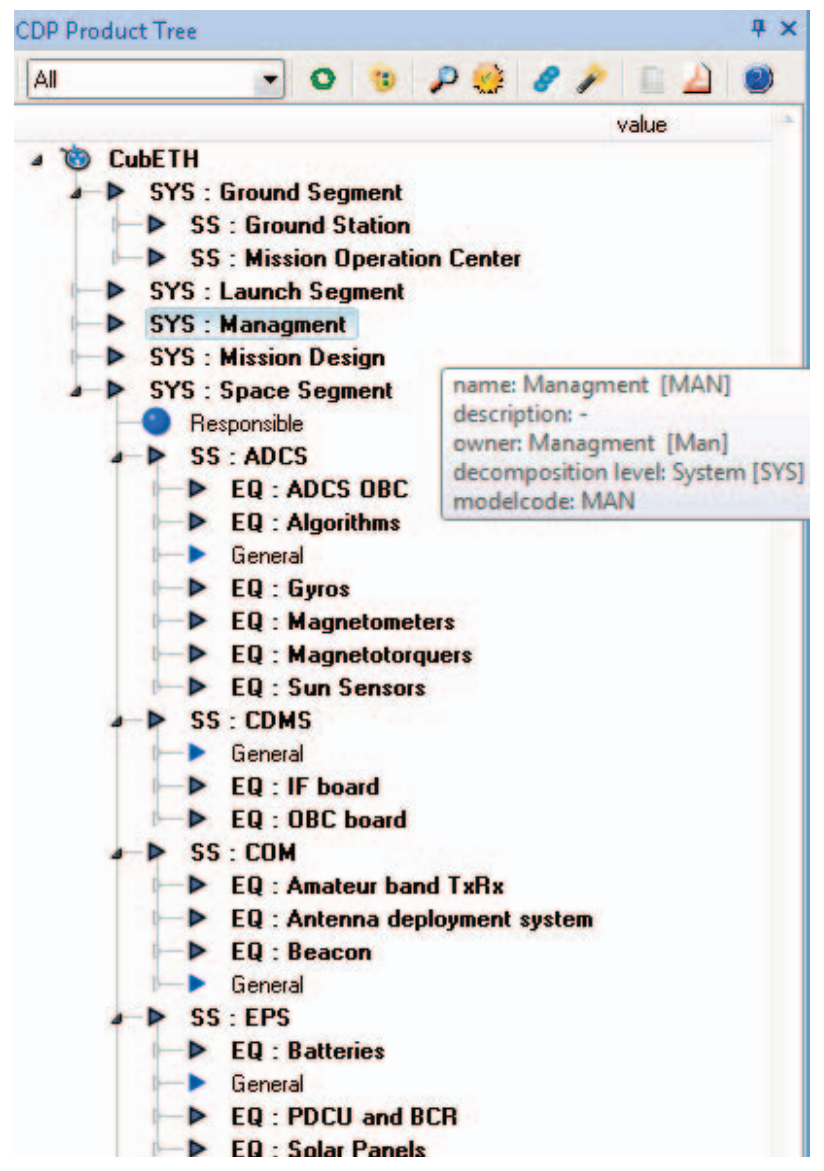

Figure 3 Example of the Product Breakdown Structure view for the CubETH nanosatellite project. This is example was done using CDP System[14].

Mission Design-Satellite ToolKit (Analytical Graphics, Inc) is used to prepare mission timeline. The most important part was to correlate Attitude Determination and Control Susbsystem (ADCS) subsystem performance with the science requirements. Extensive simulations were run to establish mission parameters. Mission scenarios were also used to evaluate all other system parameters and establish parameters for system trades.

Telecom-SwissCube heritage models are used to estimate the link budget for the mission. The goal was to significantly increase the date rate as compared to that of the SwissCube. The most effective solution was to purchase one of the existing telecom systems. During the study we have evaluated a make-it or buy-it trade and decided to go for a improve redundancy and downlink rate for the SwissCube design.

Attitude determination and control-Based on simulations and trade off analysis, SwissCube ADCS design was adopted with addition of Extended Kalman filtering for determination [15]. The team has compiled more than 6 years of flight data, which serves as the baseline to define performance characteristics for CubETH ADCS. The focus of this study is on an intensive testing and verification program for the new satellite. Now ADCS is undergoing intensive testing to verify its functionality and conformance to requirements. For following studies, $\mathrm{CDF}$ has now capability to perform trade-off analysis between different hardware architectures. Models are being developed, to allow implementation the following modes on the satellites: a) Target acquisition; b) spin-up; c) stable rotation; d) 3-axis controlled pointing.

Launch vehicle-Different options were studied. For this mission a range of orbits between 500 and $550 \mathrm{~km}$ will satisfy the requirement to deorbit the satellite within 25 years, in order to satisfy ESA space debris guidelines. Mission plan includes a possibility to perform some science observations at orbits below $200 \mathrm{~km}$, before re-entering the atmosphere.

\section{CHEOPS}

The CHaracterizing ExoPlanets Satellite (CHEOPS) will be a small satellite mission [13] (total mass < $250 \mathrm{~kg}$ ) dedicated to the search for transits by means of ultrahigh precision photometry on bright stars already known to host planets. By being able to point at nearly any location on the sky, it will provide the unique capability of determining accurate radii for a subset of those planets for which the mass has already been estimated from ground-based spectroscopic surveys. Phase A studies were performed at the EPFL Space Center between 2010 and 2012. Technical report was submitted to the PI and eventually, as a part of a larger proposal, to the Swiss Space Office and was distributed to non-technical project management.

The main science goals of the CHEOPS mission will be to study the structure of exoplanets with radii typically ranging from 1-6 $\mathrm{R}_{\text {Earth }}$ orbiting bright stars. With an accurate knowledge of masses and radii for an unprecedented sample of planets, CHEOPS will set new constraints on the structure and hence on the formation and evolution of planets in this mass range. 


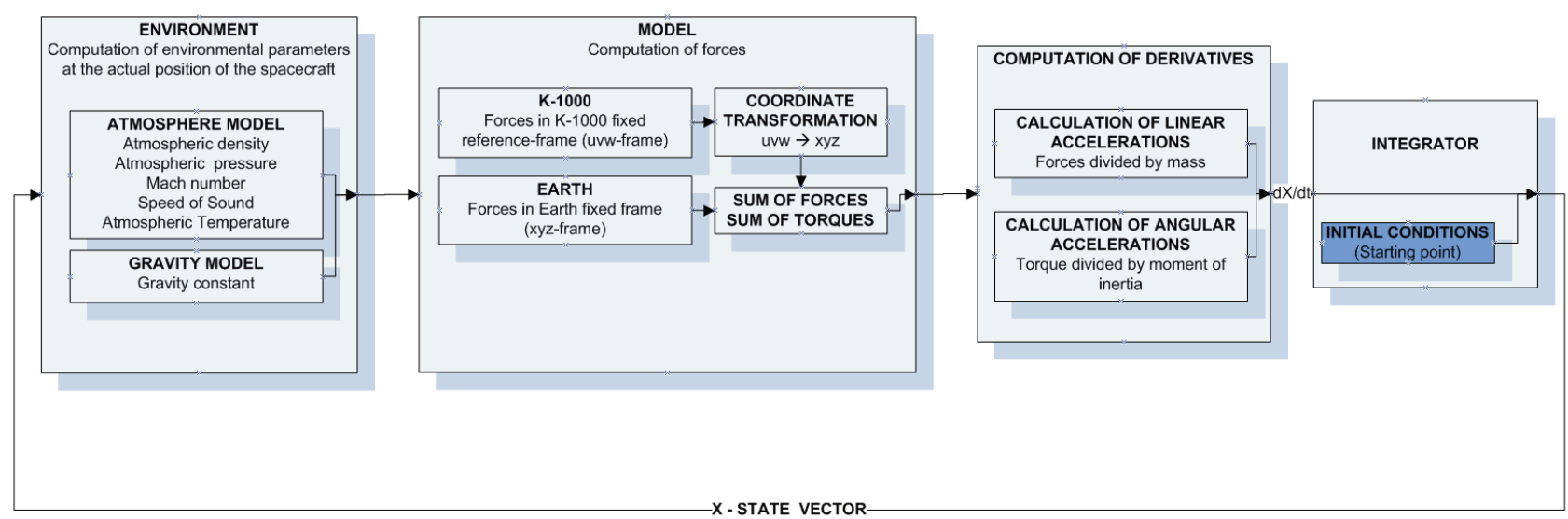

The concurrent design facility was used to establish main systems engineering budgets (mass, power, costs) and identify options for mission design. Different orbits were considered for science options (LEO up $1200 \mathrm{~km}$, GTO

highly elliptical and L2). However, all of these options were unacceptable due to costs constraints. The baseline orbit is Sun synchronous with altitude no higher than $800 \mathrm{~km}$, following the terminator $(6 \mathrm{am} / 6 \mathrm{pm}$ local time).

A new set of models was created for the CHEOPS study with the focus on already available COTS components for satellites of this size. One of goals was to evaluate "make-it or buy-it" tradeoff for the project. In the end, the decision was made to purchase an already available platform.

The results of the study made at EPFL CDF were a part of the proposal submitted to the European Space Agency for a small class mission. At the time of this writing, the mission has passed the mission adoption phase and is planned for launch in 2017.

\section{K1000}

The EPFL Space Center CDF was also used in an aerospace project. This project has implemented a complete design loop: shape/ structure - aerodynamics -mission - changes in shape (Figure 4). This project took place in the EPFL Space center between 2008 and 2010.

This project was to design a sub-orbital plane [16], in which passengers can experience the $0 \mathrm{~g}$ environment. The plane is deployed from a commercial plane at the altitude of $10 \mathrm{~km}$ and then uses its own engines to climb up to an altitude of $100 \mathrm{~km}$. After a few seconds of microgravity environment the plane glides to a safe landing. This project consisted of mechanical, aerodynamics and propulsion subsystems. A general view of the plane is shown in Figure 5. Overall plane and mission parameters were all collected in a system sheet. The team has computed aerodynamic properties of the space plane and validated primary requirements of the project.

The main focus of the study was safe return of the plane with the passengers. Acceleration loads must not exceed $5 \mathrm{~g}$.
These requirements were reflected across the system in the shape of the plane, control algorithms for descent and landing as well as operational constraints.

During this study the Concurrent Design was used for centralized model approach. In contrast with classic modeling, where subsystems models are implemented independently, MATLAB/Simulink environment (see Figure 4) integrated inputs from different submodels. Each of the subsystem engineers was responsible for contributing a model block to the system and the role of the systems engineer was to integrate all inputs. All of the important parameters are displayed in the summary Excel sheet, which is also used as the input table. Excel sheets were also used to summarize model results. This approach allowed very efficient implementation of a complex design loop, which included some very demanding computations of aerodynamic properties of the vehicle. The structural shape of the plane was designed by mechanical engineers. Using this shape, aerodynamic coefficients were computed on a cluster system. Results of aerodynamic simulation were then used for control algorithms and mission design.

Once the model was established and validated, additional studies were performed on a secondary propulsion system (for improved security of the descent and landing), some aspects of vehicle and passenger safety and prepared examples of the flight failure tree and possible outcomes. Through extensive use of visualization tools linked to the model, customer experience (for example Figure 5) was improved via better positioning of windows. 


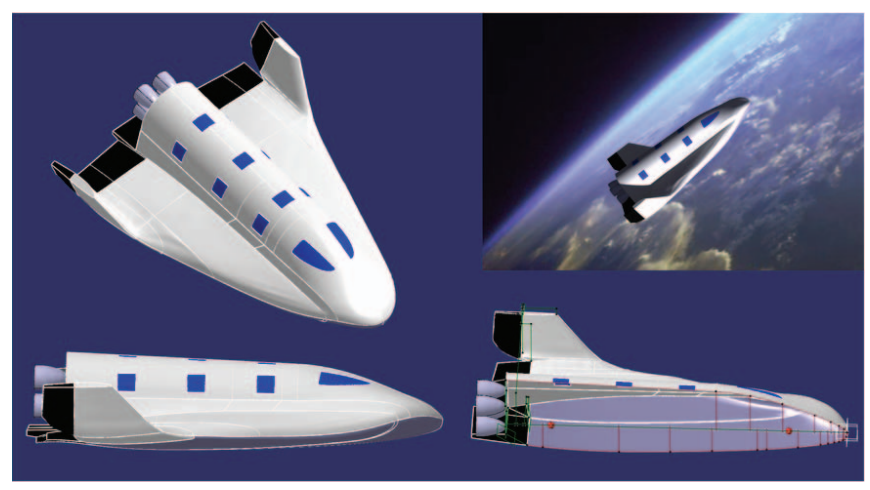

Figure 5 Computer rendering of the suborbital plane to evaluate obstacles for view from the cabin. One of the goals was to ensure adequate customer experience during the flight.

\section{Other projects}

A Hybrid motorcycle was also considered in a lab in mechanical engineering. CDF facilities were used in an ESA Technology Research project to design a small antenna for microsatellites. These projects were very focused interdisciplinary projects, which did not require a full team. For the hybrid motorcycle project, a team of engineers developed a set of models, which were completely unrelated to space. This has proven the utility of the CDF to projects that are not space related.

The SOLVE mission study was lead by the EPFL Space Center in 2015. The SOLVE study is considering a constellation of small satellites carrying innovative hyperspectral camera to study these processes at hourly timescales. This mission will contribute to monitoring natural disasters, phenology of various plants. Data from the SOLVE mission will be integrated with precision farming applications and water quality monitoring tools. These measurements will be supported by in-situ monitoring from aerial platforms (e.g. UAV or high altitude platforms). The study is funded by the Swiss Space Office of the State Secretariat for Education, Research and Innovation of the Swiss Confederation (SERI/SSO) in the framework of "Call for ideas for small missions" 2015 with support from the Swiss Space Center. This was the first study with a distributed team. Experts were situated in other laboratories and institutions. CDF concept was a great benefit for the team and allowed us to perform a more detailed study than was originally anticipated. Science team is lead by APHYS and EPSL labs of EPFL. Start-up companies are heavily involved in this study with Gamaya in charge of the payload design and ELSE SA supporting the study with their expertise in space systems engineering.

\section{Beyond the Design Phase}

Concurrent Design Facilities are usually heavily used during concept phases. An interesting aspect is to explore possibilities for using facilities in implementation phases (Phase $\mathrm{C}$ and D of a spacecraft mission project). Especially, how CDF tools and models can be used for the validation and verification process, resource management and regular planning.

Validation and verification - In the classic " $\mathrm{V}$ " pattern of the systems engineering process, the design phase is followed by implementation and design verification and validation phase. Industry standard tools, such as DOORS, usually handle requirements management, including validation and verification. Student teams usually do not have resources to work out detailed complex systems, especially when hardware delivery is needed. The systems engineering team has implemented a very simple database inside Excel to correlate results of modelling with requirements.

In the case of the CubETH project, requirements that were established during the study are now being verified during the prototyping phase. For example, all of the thermal environment requirements are being verified on the subsystem level on the Structural and Thermal model (STM). Requirements for power consumption are being verified on the Electrical Model (EM). Requirements workflow continues from the initial phase into development. Each requirement has a history, which can be very helpful to understand why the requirement exists and how it is linked to others.

Resource management-Work Breakdown Structure (WBS) tracking and analysis is continuously improved. Product breakdown Structure (PBS) can be handled very well with available tools. However, WBS presents a problem in universities due to a high turnover of student teams. For example, in one satellite project, more than 200 students can be involved in various capacities starting from simple semester projects (1 day per week engagement) to master students and to $\mathrm{PhD}$ students. Initially, Microsoft Project was used, linked with PBS and J-CDS. However it suffered from synchronization issues and manual input of information by the project manager. All requirements, action items, tasks and team management are now moved to the Redmine software [17]. It allows much better visibility into the status of the project. Students and managers enter information on their items themselves, as well providing documentations in the Wiki interface. This approach allows to use web-based tools as forum of on-going discussions and, most importantly, as a record of decisions made. This very useful especially in case of requirements, since sometimes the record on logic inside an individual statement may be lost. Comments and history change tracking allows to understand the nature of decisions taken early in the project.

\section{Challenges}

The EPFL Space Center Concurrent Design Facility operates in a student environment and is tied to the university's schedule. However, the excellent educational network in Switzerland provides access to a wide range of competences and labs, which can work on joint space 
related projects. Students come to our project from many majors: mechanical engineering, robotics, microelectronics, electrical engineering, physics. The semester is only 14 weeks, and therefore there is a strong need for a fast learning curve. It is important to prepare a work statement very clearly for each student on the team and monitor his or her progress weekly. We have developed a simple process for all students who participate in CDF activities with milestones along the way. This process encourages presentations, team discussions and document writing. Students are very efficient searching for information and updating our equipment databases for various subsystems, especially if senior students or young engineers have developed models.

For project activities with students in the space technologies program, mobility is very important. Tools installed on a particular set of computers are effective, but underused since students work at home or in other co-working environments. Further alternatives exist, to make models and databases available via a simple Web interface.

The next major step will be to upgrade the system with the Open Concurrent Design Tool (OCDT) developed by the European Space Agency. This is a modern and extendable tool based on ECSS-E-TM-10-25 format [18]. It keeps the benefits of the Integrated Data Model from previous development. The new OCDT implementation is based on open source software to the maximum extent possible and allows multi-site or distributed sessions. To simplify access to databases and models ConCORDE tool was developed for Excel. It allows easy interface to parameter and equipment databases and budget tracking. These tools are distributed under the ESA Community Open Source License.

The Space Center now developes activities to support student projects outside the space domain, especially in the framework of the new EPFL "Interdisciplinary projects" initiative. CDF will be used not only for space projects with the Space Center, but also by other faculties for multidisciplinary educational projects. Swiss industry has shown great interest in the concurrent design approach for proposal writing and we are looking towards a fruitful collaboration.

\section{SUMMARY}

The Concurrent Design Facility at the EPFL Space Center EPFL has been operational for the last five years. We have shown that the concept of Concurrent Design can be used for education in space systems engineering as well as in other major fields (mechanical engineering, microelectronics). Significant experience was gained on how to engage students and how to include CDF studies into curriculum. A process was established to develop both models (with engineers and senior students) and databases to create necessary ingredients for model based systems engineering. This system can be maintained, updated and synchronized with academic schedules. Concurrent design approach was used for research projects beyond design projects. For example, in-house student satellite projects, allow establishing a process for validation of models and verification of project requirements.

CDF concept relies on social interaction between subsystem experts. The student environment is nurturing these kinds of interactions. This is one of the key parts of engineering education at the Space Center.

\section{ACKNOWLEDGEMENTS}

This work was funded by internal EPFL funds to improve quality of education. Continuing support to the CDF was provided by the Swiss Space Center. J-CDS company (now part of the RHEA group) has provided their CDP product for academic use and we thank Arne Matthyssen, Sam Gerene and Martin Fijneman for their discussions, suggestion and support of the EPFL CDF. We are also grateful to the OCDT development team lead by Massimo Bandecchi and Hans-Peter de Konig of European Space Agency for providing us advice and support.

\section{REFERENCES}

[1] M. Bandecchi, B. Melton, and F. Ongaro, "Concurrent engineering applied to space mission assessment and design," Esa Bull. Sp. Agency, no. 99, pp. 34-40, 1999.

[2] R. Wheeler, M. Adler, and B. Sherwood, "The new team X," 2007, vol. 9, p. 6143.

[3] K. Case, "Cohesion, Power, and Flexibility: The JPL Formulation MBSE Vision."

[4] A. Braukhane, "The Dark Side of Concurrent Design - A Story of Improvisations, Workarounds, Nonsense and Success," 2014.

[5] E. Eisenamann, "Evolving a classical SRDB into an Engineering Database," 2014.

[6] M. Bonerba and J.-C. Meyer, "A cost-effective solution to improve the space systems design process: Concurrent Engineering Facility at OHB System," 2014.

[7] M. Fijnman and E. Gil, "Concurrent Engineering in Student Course Practice Space Systems Engineering."

[8] A. Golkar, "Concurrent Engineering Design Laboratory: Pioneering Concurrent Engineering in the Russian Federation,” 2014.

[9] M. Fijnman and A. Mathyssen, "Concurrent Design Applications in Construction, Offshore Industry and Engineering Education," 2010.

[10] N. Wirth, Algorithms + Data Structures = Programs. Upper Saddle River, NJ, USA: Prentice Hall PTR, 1978.

[11] S. Rossi and A. Ivanov, "Thermal model for cubesat: A simple and easy model from the Swisscube's thermal flight data," in Proceedings of the International Astronautical Congress, IAC, 2013, vol. 13, pp. 9919-9928. 
[12] A. Ivanov, C. Hollenstein, M. Rothacher, M. Klaper, J. Wassner, H. Mathis, P. Fleischman, B. Mannel, F. Belloni, L. Masson, R. Wiesendanger, S. Rossi, and A. Consoli, "CubETH: Low Cost GNSS Space Experiment for Precise Orbit Determination.," 2014.

[13] C. Broeg, A. Fortier, D. Ehrenreich, Y. Alibert, W. Baumjohann, W. Benz, M. Deleuil, M. Gillon, A. Ivanov, R. Liseau, M. Meyer, G. Oloffson, I. Pagano, G. Piotto, D. Pollacco, D. Queloz, R. Ragazzoni, E. Renotte, M. Steller, and N. Thomas, "CHEOPS: A transit photometry mission for ESA's small mission programme," EPJ Web Conf., vol. 47, p. 03005, Apr. 2013.

[14] M. Fijneman, "Concurrent Design Applications in Construction, Offshore Industry and Engineering Education," SECESA 2010, 2010.

[15] A. B. Ivanov, L. Masson, S. Rossi, F. Belloni, N. Mullin, R. Wiesendanger, M. Rothacher, C. Hollenstein, B. Mannel, D. Willi, M. Fisler, P. Fleischman, H. Mathis, M. Klaper, M. Joss, and E. Styger, "CUBETH: NANO-SATELLITE MISSION FOR ORBIT AND ATTITUDE DETERMINATION USING LOW-COST GNSS RECEIVERS," in 66th International Astronautical Congress, 2015.

[16] Wi. Pierre, P. Leyland, J. Pascal, W. Reto, I. Anton, P. Coue, L. Gathier, L. Francois, and D. S. P. Philippe, "Integrative Design Loop for the Preliminary Study of a Suborbital Transportation System," American Institute of Aeronautics and Astronautics.

[17] “Redmine.".http://redmine.org

[18] "ECSS-E-TM-10-25:Engineering design model data exchange (CDF)." European Cooperation for space standardization.

\section{BIOGRAPHY}

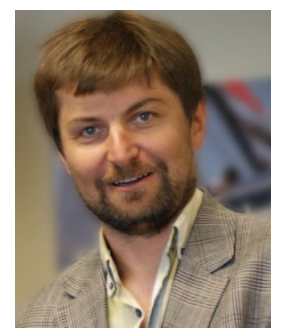

Dr. Anton Ivanov is a scientist with the EPFL Space Center (eSpace) in Lausanne Switzerland. He is the project manager for the CubETH Cubesat project, study leader for the CHEOPS satellite and is responsible for the Minor in Space Technologies. After receiving his $P h D$ in Planetary Science from Caltech in 2000, Dr. Ivanov joined the Jet Propulsion Laboratory to contribute to Mars Global Surveyor, Mars Odyssey, Mars Express and Mars Science Laboratory projects. In 2007, Dr. Ivanov joined Swiss Space Center to lead development of the Concurrent Design Facility.

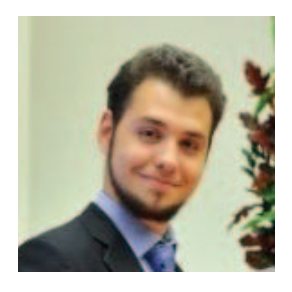

Louis Masson has graduated as a engineer in microelectronics specialized in robotics and autonomous systems in 2013 at the EPFL. He did his Master thesis at the Swiss Space Center developing the onboard computer of the CubETH Cubesat project, and has since joined the CubETH systems engineering team at the Swiss Space Center.

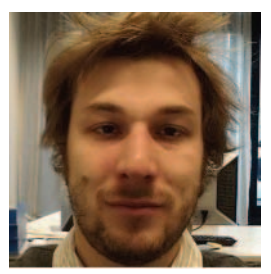

Federico Belloni is an electrical system engineer at the Swiss Space Center in Lausanne Switzerland. He is the electrical system engineering for the CubETH CubeSat project and responsible for the electronics designs at the Swiss Space Center. After receiving his HES engineer degree in Telecommunication from HEIG-VD in 2007 and Master in Electronics from EPFL in 2011, he joined the Swiss Space Center to contribute to CHEOPS, CubETH, CleanSpaceOne, Cadre and SpaceCam projects. 
